\title{
Global Groundwater Sustainability
}

\author{
This is a non-peer reviewed preprint submitted to EarthArXiv which is in review at "Groundwater" as \\ a Guest Editorial
}

\author{
Tom Gleeson, \\ Department of Civil Engineering and School of Earth and Ocean Sciences, \\ University of Victoria, Canada \\ tgleeson@uvic.ca
}

Groundwater resources are the most reliable source of freshwater on the planet, so long as they are sustainably managed. While serious groundwater depletion and contamination are well documented in several regions around the world, other regions have the potential to leverage under-developed groundwater resources to fuel local human development. Here, I argue for the importance of global perspectives in groundwater governance and management, introduce a recent global groundwater sustainability initiative, and critically reflect on the role of hydrogeologists in groundwater sustainability.

Some hydrogeologists reasonably question the appropriate scale to assess groundwater problems: are groundwater resources, governance and management a global problem (Konikow \& Kendy, 2005), or are groundwater problems best examined and solved at local-to-regional scales? On one hand, groundwater resources and their sustainability will always depend on local hydrology, politics, law, and culture (Foster et al. 2013), yet a number of arguments highlight why a global perspective on groundwater is useful (Gleeson et al. 2020):

1) better informing water governance and management for large and often transboundary aquifers in an increasingly globalized world that trades virtual water and other groundwaterdependent resources in the food-energy-water nexus;

2) systematically analyzing problems and solutions globally could enable prioritization of regions or knowledge transfer between regions;

3) creating visualizations and interactive opportunities that are consistent across the globe to improve understanding and appreciation of groundwater resources;

4) emphasizing the role of groundwater in global sustainability frameworks such as the UN Sustainable Development Goals and planetary boundaries;

5) understanding and quantifying the interactions between groundwater and other components of the Earth System; and

6) building local to global networks of interdisciplinary experts and practitioners to support sustainable solution development and application.

Groundwater is simultaneously a local, regional and increasing global resource and examining groundwater problems, solutions and interactions at all scales is crucial. However, it is nonetheless challenging to think simultaneously across multiple scales: can a local aquifer budget consider the twoway feedbacks between the aquifer and global environmental change and global food systems?

To encourage a global perspective and draw global attention and commitment for more action on groundwater, a number of scientists, practitioners and experts recently discussed, drafted and signed the Global Groundwater Sustainability Statement (www.groundwaterstatement.org). The Statement has gained great momentum, like a stream gaining baseflow from many aquifers, thanks to myriad contributors including the leadership team (on whose behalf I am writing), the communications team, 
the website team and importantly the over 1100 signatories from over 90 countries. To have greater resonance, we wrote a compelling message to a broad audience in plain language. Instead of discussing heterogeneous aquifer properties or the complexity of model boundary conditions, we focused our message on groundwater's utility in achieving the UN Sustainable Development Goals and in climate change adaptation strategies. The Statement brought various communities together from around the world and such community-building and ally-finding are important - we are not alone in acknowledging a problem and being motivated to make change.

Society, funding agencies, and academic organizations are all advocating for more socially relevant and impactful science. It is crucial for scientists to continue contributing robust, objective analyses and recommendations, which are our foundational contribution to society. But we can be more relevant and impactful by also contributing to policy briefs and science communication, organizing workshops, and working with water managers, decision makers and elected officials to apply science to real-world problems. As one of many initiatives, you can support or use the Statement in a number of ways:

- Sign and spread the word through email, social media or writing an opinion/commentary article for a local or national newspaper;

- Use the broad support of the Statement to support regional, state, or national groundwater management and policy initiatives; and

- Connect with like-minded scientists and practitioners in your region or nation to further the objectives of the Statement such as developing and implementing groundwater sustainability plans for aquifers.

Groundwater scientists, practitioners and experts have a deep understanding of groundwater problems as well as an important role in developing solutions to groundwater sustainability challenges. If we don't raise these issues and contribute to these conversations, who will?

Acknowledgements: Written on behalf of the Statement leadership team that includes Karen Villholth, Richard Taylor, Deb Perrone, Jay Famiglietti, David Hyndman, Anne van Loon and Yoshi Wada.

\section{References}

Foster, S., Chilton, J., Nijsten, G.-J., \& Richts, A. (2013). Groundwater - a global focus on the 'local resource.' Current Opinion in Environmental Sustainability, 5(6), 685-695.

doi.org/10.1016/j.cosust.2013.10.010

Gleeson, T., Cuthbert,M., Ferguson,G., and Perrone, D. (2020) Global Groundwater Sustainability, Resources, and Systems in the Anthropocene. Annu. Rev. Earth Planet. Sci. 48:X-X doi.org/10.1146/annurev-earth-071719-055251

Konikow, L. F., \& Kendy, E. (2005). Groundwater depletion: a global problem. Hydrogeology Journal, 13(1), 317-320. doi.org/10.1007/s10040-004-0411-8 